\title{
Iterative Solution for Systems of a Class of Abstract Operator Equations in Banach Spaces and Application
}

\author{
Hua Su \\ School of Mathematics and Quantitative Economics, Shandong University of Finance and Economics, Jinan, \\ Shandong 250014, China \\ Correspondence should be addressed to Hua Su; jnsuhua@163.com \\ Received 3 June 2020; Accepted 26 June 2020; Published 13 July 2020 \\ Guest Editor: Chuanjun Chen \\ Copyright (c) 2020 Hua Su. This is an open access article distributed under the Creative Commons Attribution License, which \\ permits unrestricted use, distribution, and reproduction in any medium, provided the original work is properly cited. \\ In this paper, by using the partial order method, the existence and uniqueness of a solution for systems of a class of abstract \\ operator equations in Banach spaces are discussed. The result obtained in this paper improves and unifies many recent results. \\ Two applications to the system of nonlinear differential equations and the systems of nonlinear differential equations in Banach \\ spaces are given, and the unique solution and interactive sequences which converge the unique solution and the error estimation \\ are obtained.
}

\section{Introduction}

Guo and Lakshmikantham [1] introduced the definition of the mixed monotone operator and the coupled fixed point, and there are many good results (see [2-23]). Recently, from paper [6], using the monotone iterative techniques, the iterative unique solution of the following nonlinear mixed monotone Fredholm-type integral equations in Banach spaces $E$ is obtained:

$$
u(t)=\int_{I} H(t, s, u(s)) \mathrm{d} s, \quad I=[a, b],
$$

where $I=[a, b]$ and $H \in C[I \times I \times E, E]$.

In this paper, the following nonlinear abstract operator equations in Banach spaces $E$ are discussed:

$$
\left\{\begin{array}{l}
u=A(u, v) \\
v=B(v, u)
\end{array}\right.
$$

where $A, B: D \times D \longrightarrow E$ and $D$ is a partial interval in $E$ which is denoted as the following:

$$
D \doteq\left[u_{0}, v_{0}\right] \equiv\left\{u \in E \mid u_{0} \leq u \leq v_{0}\right\} .
$$

For convenience, the following assumptions are made:
$\left(H_{1}\right)$ There exist positive bounded operators $T_{i}: E \longrightarrow E(i=1,2)$ which satisfy.

$\left(I+T_{1}-T_{2}\right) x \geq \theta \Longrightarrow x \in P$, and for any $u_{i}, v_{i} \in D(i=1,2), u_{1} \leq u_{2}, v_{1} \leq v_{2}$, the following is obtained:

$B\left(v_{2}, u_{1}\right)-B\left(v_{1}, u_{2}\right) \geq-T_{1}\left(v_{2}-v_{1}\right)-T_{2}\left(u_{2}-u_{1}\right)$,
$A\left(v_{2}, u_{1}\right)-A\left(v_{1}, u_{2}\right) \geq-T_{1}\left(v_{2}-v_{1}\right)-T_{2}\left(u_{2}-u_{1}\right)$.

$\left(H_{2}\right) u_{0}+T_{2}\left(v_{0}-u_{0}\right) \leq A\left(u_{0}, v_{0}\right), \quad B\left(v_{0}, u_{0}\right) \leq v_{0}-T_{2}$ $\left(v_{0}-u_{0}\right)$.

$\left(H_{3}\right)$ There exists a positive bounded operator $L: E \longrightarrow E$, and for any $u, v \in D, u \leq v$, the following is obtained:

$-\left(T_{1}+T_{2}\right)(v-u) \leq B(v, u)-A(u, v) \leq L(v-u)$.

$\left(H_{4}\right) L T_{2}=T_{2} L, L T_{1}=T_{1} L, T_{1} T_{2}=T_{2} T_{1}$ in which the spectral radius satisfies 


$$
\begin{gathered}
r(L)+r\left(T_{1}\right)+r\left(T_{2}\right)<\inf \left\{|\lambda|: \lambda \in \sigma\left(I+T_{1}-T_{2}\right)\right\}, \\
r\left(T_{1}-T_{2}\right)<1 .
\end{gathered}
$$

In this paper, firstly, by using the partial order method, the existence and uniqueness of a solution for systems of a class of abstract operator equations in Banach spaces are discussed. And next, two applications to the system of nonlinear integral equations and the system of nonlinear differential equations in Banach spaces are given, and the unique solution and interactive sequences which converge a unique solution and the error estimation are obtained.

\section{The Interactive Solution of Abstract Operator Equations}

Let $P$ be a cone in $E$, i.e., a closed convex subset, such that $\lambda P \subset P$ for any $\lambda \geq 0$ and $P \cap\{-P\}=\{\theta\}$. A partial order $\leq$ in $P$ is defined as $x \leq y \Leftrightarrow y-x \in P$. A cone $P$ is said to be normal if there exists a constant $N>0$ which satisfies $x, y \in E, \theta \leq x \leq y$, implying $\|x\| \leq N\|y\|$, where $\theta$ denotes the zero element of $E$. And, the smallest number $N$ is called as the normal constant of $P$ and denoted as $N_{P}$. The cone $P$ is normal iff every ordered interval $[x, y]=\{z \in E: x \leq z \leq y\}$ is bounded.

The following theorem is the main results in this section.

Theorem 1. Let $P$ be a cone in $E, u_{0}, v_{0} \in E, u_{0} \leq v_{0}$. Suppose that $A, B: D \times D \longrightarrow E$ satisfies conditions $\left(H_{1}\right)-\left(H_{4}\right)$. Then,

(i) There exists a unique solution of equation (2) $\left(u^{*}, u^{*}\right)$ in $D \times D$, and for any solutions of equation (2) $(u, u) \in D \times D$, one has $u=u^{*}$.

(ii) For any initial value $x_{0}, y_{0} \in D, x_{0} \leq y_{0}$, the following iterative sequences are constructed:

$$
\left\{\begin{array}{l}
x_{n}=\left(I+T_{1}-T_{2}\right)^{-1}\left[A\left(x_{n-1}, y_{n-1}\right)+T_{1} x_{n-1}-T_{2} y_{n-1}\right], \\
y_{n}=\left(I+T_{1}-T_{2}\right)^{-1}\left[B\left(y_{n-1}, x_{n-1}\right)+T_{1} y_{n-1}-T_{2} x_{n-1}\right],
\end{array}\right.
$$

which satisfy $\left\|x_{n}-u^{*}\right\| \longrightarrow 0,\left\|y_{n}-u^{*}\right\| \longrightarrow 0(n \longrightarrow \infty)$, and for any $\delta$,

$$
\frac{r(L)+r\left(T_{1}\right)+r\left(T_{2}\right)}{\inf \left\{|\lambda|: \lambda \in \sigma\left(I+T_{1}-t_{2}\right)\right\}}<\delta<1,
$$

there exists a natural number $n_{0}$ which satisfies as $n \geq n_{0}$, the following is obtained:

$$
\left\{\begin{array}{l}
\left\|x_{n}-u^{*}\right\| \leq 2 N_{p} \delta^{n}\left\|v_{0}-u_{0}\right\| \\
\left\|y_{n}-u^{*}\right\| \leq 2 N_{p} \delta^{n}\left\|v_{0}-u_{0}\right\| .
\end{array}\right.
$$

Proof. By $r\left(T_{1}-T_{2}\right)<1$, it is known that the operator $(I+$ $\left.T_{1}-T_{2}\right)$ is reversible. And, from condition $\left(H_{1}\right)$, $\left(I+T_{1}-T_{2}\right)^{-1}$ is the positive operator. Let

$$
\left\{\begin{array}{l}
F(u, v)=\left(I+T_{1}-T_{2}\right)^{-1}\left[A(u, v)+T_{1} u-T_{2} v\right], \\
G(v, u)=\left(I+T_{1}-T_{2}\right)^{-1}\left[B(v, u)+T_{1} v-T_{2} u\right] .
\end{array}\right.
$$

Then, equation (7) can be substituted by the following:

$$
\left\{\begin{array}{l}
x_{n}=F\left(x_{n-1}, y_{n-1}\right), \\
y_{n}=G\left(y_{n-1}, x_{n-1}\right) .
\end{array}\right.
$$

By conditions $\left(H_{1}\right)-\left(H_{3}\right)$, it is easy to obtain that operators $F$ and $G$ satisfy the following:

(1) $u_{0} \leq F\left(u_{0}, v_{0}\right) \leq G\left(v_{0}, u_{0}\right) \leq v_{0}$

(2) $F, G: D \times D \longrightarrow E$ are the mixed monotone operator

(3) $\theta \leq G(v, u)-F(u, v) \leq H(v-u), u_{0} \leq u \leq v \leq v_{0}$, where $H=\left(L+T_{1}+T_{2}\right)\left(I+T_{1}-T_{2}\right)^{-1}$

Letting $\quad u_{n}=F\left(u_{n-1}, v_{n-1}\right)$ and $v_{n}=G\left(v_{n-1}, u_{n-1}\right)$ $(n=1,2, \ldots)$, the following two results are obtained by mathematical induction:

$$
\begin{aligned}
& u_{0} \leq u_{1} \leq \cdots \leq u_{n} \leq \cdots \leq v_{n} \leq \cdots \leq v_{1} \leq v_{0}, \\
& u_{n} \leq x_{n} \leq y_{n} \leq v_{n}, \\
& \theta \leq v_{n}-u_{n} \leq H^{n}\left(v_{0}-u_{0}\right), \quad n=1,2, \ldots
\end{aligned}
$$

In fact, from (1) and (3), one has

$$
\begin{aligned}
u_{0} & \leq u_{1} \leq v_{1} \leq v_{0}, \\
u_{1} & \leq x_{1} \leq y_{1} \leq v_{1}, \\
0 & \leq v_{1}-u_{1} \leq H\left(v_{0}-u_{0}\right) .
\end{aligned}
$$

Suppose that for $n=k$, one has (12) and (13). Then, as $n=k+1$, by (2) and (3), the following is obtained:

$$
\begin{aligned}
u_{k+1} & =F\left(u_{k}, v_{k}\right) \leq x_{k+1}=F\left(x_{k}, y_{k}\right) \leq G\left(y_{k}, x_{k}\right)=y_{k+1} \\
& \leq G\left(v_{k}, u_{k}\right)=v_{k+1}, \\
\theta & \leq v_{k+1}-u_{k+1}=G\left(v_{k}, u_{k}\right)-F\left(u_{k}, v_{k}\right) \leq H\left(v_{k}-u_{k}\right) \\
& \leq H^{k+1}\left(v_{k}-u_{k}\right) .
\end{aligned}
$$

Then, it is known that

$$
\begin{gathered}
u_{k} \leq u_{k+1} \leq x_{k+1} \leq y_{k+1} \leq x_{k+1} \leq v_{k}, \\
\theta \leq v_{k+1}-u_{k+1} \leq H^{k+1}\left(v_{k}-u_{k}\right) .
\end{gathered}
$$

Then, for any natural number $n,(12)$ and (13) are obtained by mathematical induction.

Next, it is proved that $\left\{x_{n}\right\}$ is Cauchy sequences. From condition $\left(H_{4}\right)$, it is known that

$$
\left(L+T_{1}+T_{2}\right)\left(I+T_{1}-T_{2}\right)^{-1}=\left(I+T_{1}-T_{2}\right)^{-1}\left(L+T_{1}+T_{2}\right),
$$

then by ([14], V 3.9), $r(H) \leq\left[r(L)+r\left(T_{1}\right)+r\left(T_{2}\right)\right]$ $\mathrm{r}\left[\left\{\left(\mathrm{I}+\left\{\mathrm{T}_{-}\{\{1\}\}\right\}-\left\{\mathrm{T}_{-}\{\{2\}\}\right\}\right)^{\wedge}\{\{-1\}\}\right\}\right]$.

Thus, for any $\delta:\left(r(\bar{L})+r\left(T_{1}\right)+r\left(T_{2}\right)\right) /(\inf \{|\lambda|: \lambda \epsilon$ $\left.\left.\sigma\left(I+T_{1}-t_{2}\right)\right\}\right)<\delta<1$, the following is obtained: 


$$
\begin{aligned}
\lim \left\|H^{n}\right\|^{1 / n}= & r(H) \leq\left[r(L)+r\left(T_{1}\right)+r\left(T_{2}\right)\right] r \\
& \cdot\left[\left(I+T_{1}-T_{2}\right)^{-1}\right] \\
= & \frac{\left[r(L)+r\left(T_{1}\right)+r\left(T_{2}\right)\right]}{\inf \left\{|\lambda|: \lambda \in \sigma\left(I+T_{1}-t_{2}\right)\right\}}<\delta<1 .
\end{aligned}
$$

Then, there exists a natural number $n_{0}$ which satisfies

$$
\left\|H^{n}\right\| \leq \delta^{n}, \quad \forall n \geq n_{0} .
$$

And, by (12) and (13), it is obtained that

$$
\begin{aligned}
& \theta \leq u_{n} \leq u_{n+p} \leq x_{n+p} \leq y_{n+p} \leq v_{n+p} \leq v_{n}, \\
& \theta \leq u_{n} \leq x_{n} \leq y_{n} \leq v_{n}, n, \quad p=1,2, \ldots
\end{aligned}
$$

So, by (13), it is known that

$$
\begin{aligned}
& \theta \leq x_{n+p}-u_{n} \leq v_{n}-u_{n} \leq H^{n}\left(v_{0}-u_{0}\right), \\
& \theta \leq x_{n}-u_{n} \leq H^{n}\left(v_{0}-u_{0}\right) .
\end{aligned}
$$

Then, by the normality of $P$ and (19), it is known that

$$
\begin{array}{r}
\left\|x_{n+p}-u_{n}\right\| \leq N_{p}\left\|H^{n}\left(v_{0}-u_{0}\right)\right\| \leq N_{p} \delta^{n}\left\|v_{0}-u_{0}\right\|, \\
\left\|x_{n}-u_{n}\right\| \leq N_{p}\left\|H^{n}\left(v_{0}-u_{0}\right)\right\| \leq N_{p} \delta^{n}\left\|v_{0}-u_{0}\right\|, \\
n \geq n_{0}, p=1,2, \ldots
\end{array}
$$

Thus, the following is obtained:

$$
\begin{aligned}
\left\|x_{n+p}-x_{n}\right\| \leq\left\|x_{n+p}-u_{n}\right\|+\left\|x_{n}-u_{n}\right\| & \leq 2 N_{p} \delta^{n}\left\|v_{0}-u_{0}\right\|, \\
n & \geq n_{0}, p=1,2, \ldots,
\end{aligned}
$$

i.e., $\left\{x_{n}\right\}$ is Cauchy sequences. So, there exists $u^{*} \in D(D$ is bounded), such that $\lim _{n \longrightarrow \infty} x_{n}=u^{*}$.

And, by $\theta \leq y_{n}-x_{n} \leq v_{n}-u_{n} \leq H^{n}\left(v_{0}-u_{0}\right)$, the normality of $P$, and (19), one obtains

$$
\left\|y_{n}-x_{n}\right\| \leq N_{p} \delta^{n}\left\|v_{0}-u_{0}\right\|,
$$

therefore

$$
\lim _{n \longrightarrow \infty} y_{n}=u^{*}=\lim _{n \longrightarrow \infty} x_{n}, \quad x_{n} \leq u^{*} \leq y_{n}, n=1,2, \ldots
$$

Thus, $\quad\left\|x_{n}-u_{n}\right\| \leq N_{p} \delta^{n}\left\|v_{0}-u_{0}\right\|, \quad\left\|v_{n}-x_{n}\right\| \leq N_{p} \delta^{n}$ $\left\|v_{0}-u_{0}\right\|$, and

$$
\begin{gathered}
\lim _{n \longrightarrow \infty} u_{n}=u^{*}=\lim _{n \longrightarrow \infty} v_{n}, \\
u_{n} \leq u^{*} \leq v_{n}, \quad n=1,2, \ldots,
\end{gathered}
$$

so by (2), (3), and (11), it is also obtained that

$u_{n}=F\left(u_{n-1}, v_{n-1}\right) \leq F\left(u^{*}, u^{*}\right) \leq G\left(u^{*}, u^{*}\right) \leq G\left(v_{n-1}, u_{n-1}\right)=v_{n}$.
Letting $n \longrightarrow \infty \quad$ and by $(27), \quad F\left(u^{*}, u^{*}\right)=$ $G\left(u^{*}, u^{*}\right)=u^{*}$.

Then, by the definition of $F$ and $G$, one obtains $u^{*}=A\left(u^{*}, u^{*}\right), u^{*}=B\left(u^{*}, u^{*}\right)$, i.e., $\left(u^{*}, u^{*}\right)$ is a solution of equation (2).

Lastly, it is proven that the solution is unique. Supposing that $(u, u) \in D \times D$ also satisfies equation (2), then by (11) and mathematical induction, the following is obtained:

$$
u_{n} \leq u \leq v_{n}, \quad(n=1,2, \ldots) .
$$

Thus, $u=u^{*}$.

And, letting $p \longrightarrow \infty$ in (24), as $n \geq n_{0}$, the following is obtained:

$$
\left\|x_{n}-u^{*}\right\| \leq 2 N_{p} \delta^{n}\left\|v_{0}-u_{0}\right\| .
$$

Similarly, as $n \geq n_{0}$, the following is obtained:

$$
\left\|y_{n}-u^{*}\right\| \leq 2 N_{p} \delta^{n}\left\|v_{0}-u_{0}\right\| .
$$

The proof is complete.

Remark 1. In Theorem 1, it is only supposed that operators $A$ and $B$ satisfy the partial condition, and the unique solution and interactive sequences which converge a unique solution are obtained.

\section{The Application of Nonlinear Integral Equations}

In this section, the following nonlinear integral equations are considered:

$$
\left\{\begin{array}{l}
u(t)=f_{1}(t, u(t), v(t))+\int_{0}^{t} g_{1}(s, u(s), v(s)) \mathrm{d} s, \\
v(t)=f_{2}(t, v(t), u(t))+\int_{0}^{t} g_{2}(s, u(s), u(s)) \mathrm{d} s
\end{array}\right.
$$

where $f_{i} \in\left[I \times \mathbf{R}_{+} \times R_{+}, R_{+}\right]$(here, the continuity of $f_{i}$ is not assumed) and $g_{i} \in C\left[I \times \mathbf{R}_{+} \times \mathbf{R}_{+}, \mathbf{R}_{+}\right], \quad i=1,2$, $I=[0,+\infty)$, and $E$ is a real Banach space with norm $\|\cdot\|$.

In this section, the iterative solution of a nonlinear integral equation (33) is discussed. For convenience, the following assumptions are made:

$\left(L_{1}\right)$ For the nonnegative bounded continuous function $a(t), b(t)$, and nonnegative integrable $c(t), d(t)$, one has

$$
\begin{aligned}
& f_{2}(t, u, \theta) \leq a(t) u+b(t), \\
& g_{2}(t, u, \theta) \leq c(t) u+d(t) .
\end{aligned}
$$

$\left(L_{2}\right)$ There exists a constant $M>0$, for any $u, v \in E, u \leq v$, which satisfies

$$
\begin{aligned}
& f_{i}(t, v, u)-f_{i}(t, u, v) \geq-M(v-u), \\
& g_{i}(t, v, u)-g_{i}(t, u, v) \geq 0, \quad(i=1,2) .
\end{aligned}
$$

$\left(L_{3}\right)$ For any $u, v \in E, u \leq v$, the following is satisfied: 


$$
\begin{gathered}
-M(v-u) \leq f_{2}(t, v, u)-f_{1}(t, u, v) \leq c(t)(v-u), \\
0 \leq g_{2}(t, v, u)-g_{1}(t, u, v) \leq a(t)(v-u) . \\
\left(L_{4}\right) \max _{t \in I} a(t)<1 .
\end{gathered}
$$

In this section, the following main theorem is obtained.

Theorem 2. Let $P$ be a normal cone in E. Suppose conditions $\left(L_{1}\right)-\left(L_{4}\right)$ hold. Then, there exists a unique solution of equation (2) $\left(u^{*}, u^{*}\right) \in(E \times E)$, and there are iterative sequences converging to the unique solution, and corresponding error estimates are given.

Proof. Let $E=C[I, R]$. Then, $P_{c}=\{x \in C[I, R] \mid x(t) \geq 0$, $\forall t \in I\}$ is a cone. Thus, by the normal of $P, P_{c}$ is also normal.

The following operator is considered:

$$
\begin{aligned}
& A=F_{1}+G_{1}, \\
& B=F_{2}+G_{2},
\end{aligned}
$$

where for any $u, v \in P_{c}, t \in I$,

$$
\begin{aligned}
& F_{1}(u, v)=f_{1}(t, u(t), v(t)), \\
& G_{1}(u, v)=\int_{0}^{t} g_{1}(s, u(s), v(s)) \mathrm{d} s, \\
& F_{2}(v, u)=f_{2}(t, u(t), u(t)), \\
& G_{1}(v, u)=\int_{0}^{t} g_{2}(s, v(s), u(s)) \mathrm{d} s .
\end{aligned}
$$

Then, $A, B: P_{c} \times P_{c} \longrightarrow E$. It is easy to know that $\left(u^{*}, u^{*}\right) \in P_{c} \times P_{c}$ is a solution of (33) if and only if $\left(u^{*}, u^{*}\right)$ is a solution of the following integral equations:

$$
\left\{\begin{array}{l}
u=A(u, v), \\
v=B(v, u) .
\end{array}\right.
$$

Next, from conditions $\left(L_{1}\right)-\left(L_{4}\right)$, it is obtained that the operators $A$ and $B$ satisfy the whole condition of Theorem 1.

In fact, $\forall u_{1}, u_{2}, v_{1}, v_{2} \in P_{c}, u_{1} \leq u_{2}, v_{1} \leq v_{2}$ :

(i) Let

$$
\begin{aligned}
L u & =a(t) u+\int_{0}^{t} c(s) u(s) \mathrm{d} s, \\
h & =b(t)+\int_{0}^{t} d(s) \mathrm{d} s, \quad t \in I, \\
L_{1} u & =a(t) u, \\
L_{2} u & =\int_{0}^{t} c(s) u(s) \mathrm{d} s .
\end{aligned}
$$

Then, $L_{1} L_{2}=L_{2} L_{1}$ and $r\left(L_{1}\right)=\max _{t \in I} a(t), r\left(L_{2}\right)=0$. Thus,

$$
r(L)=r\left(L_{1}+L_{2}\right) \leq r\left(L_{1}\right)+r\left(L_{2}\right)=\max _{t \in I} a(t)<1 .
$$

Therefore, for the equation $(I-L) u=h$, there exists a unique solution $v_{0}=(I-L)^{-1} h=\sum_{n=0}^{\infty} L^{n} h \in P$. Then, by $\left(L_{1}\right)$, for any $t \in I$, the following is obtained:

$$
\begin{aligned}
B\left(v_{0}, \theta\right)= & F_{2}\left(v_{0}, \theta\right)+G_{2}\left(v_{0}, \theta\right)=f_{2}\left(t, v_{0}(t), \theta\right) \\
& +\int_{0}^{t} g_{2}\left(s, v_{0}(s), \theta\right) \mathrm{d} s \\
\leq & a(t) v_{0}+\int_{0}^{t} c(s) v_{0}(s) \mathrm{d} s+b(t) \\
& +\int_{0}^{t} d(s) \mathrm{d} s=L v_{0}+h=v_{0} .
\end{aligned}
$$

Obviously, $\theta \leq f_{1}\left(t, \theta, v_{0}(t)\right)+\int_{0}^{t} g_{1}\left(s, \theta, v_{0}(s)\right) d=$ $A\left(\theta, v_{0}\right)$.

(ii) By $\left(L_{2}\right)$, the following is obtained:

$$
\begin{aligned}
& B\left(v_{2}, u_{1}\right)-B\left(v_{1}, u_{2}\right) \\
& \quad=f_{2}\left(t, v_{2}(t), u_{1}(t)-f_{2}\left(t, v_{1}(t), u_{2}(t)\right)\right. \\
& \quad+\int_{0}^{t}\left[g_{2}\left(s, v_{2}(s), u_{1}(s)\right)-g_{2}\left(s, v_{1}(s), u_{2}(s)\right)\right] \mathrm{d} s \\
& \quad \geq f_{2}\left(t, v_{2}(t), u_{1}(t)-f_{2}\left(t, v_{1}(t), u_{2}(t)\right) \geq-M\left(v_{2}-v_{1}\right) .\right.
\end{aligned}
$$

Similarly, $A\left(v_{2}, u_{1}\right)-A\left(v_{1}, u_{2}\right) \geq-M\left(v_{2}-v_{1}\right)$.

(iii) From $\left(L_{3}\right)$ and $\left(L_{4}\right)$, the following is obtained:

$$
\begin{aligned}
& B(v, u)-A(u, v) \\
& \quad=f_{2}(t, v(t), u(t))-f_{1}(t, v(t), u(t))+\int_{0}^{t}\left[g_{2}\left((s, v(s), u(s))-g_{1}(s, v(s), u(s))\right)\right] \mathrm{d} s \\
& \quad \geq-M(v-u)+\int_{0}^{t}\left[g_{2}(s, v(s), u(s))-g_{1}(s, v(s), u(s))\right] \mathrm{d} s \geq-M(v-u), \\
& B(v, u)-A(u, v) \\
& \quad=f_{2}(t, v(t), u(t))-f_{1}(t, v(t), u(t))+\int_{0}^{t}\left[g_{2}(s, v(s), u(s))-g_{1}(s, v(s), u(s))\right] \mathrm{d} s \\
& \quad \leq a(t)(v-u)+\int_{0}^{t} c(s)(v-u) \mathrm{d} s=L(v-u) .
\end{aligned}
$$


Then, by (41), it is known that

$-M(v-u) \leq B(v, u)-A(u, v) \leq L(v-u), \quad r(L)<1$.

Therefore, from (i), (ii), and (iii), letting $T_{1}=M_{1} I, T_{2}=0$ in Theorem 1 , it is easy to know that the condition $\left(\mathrm{H}_{4}\right)$ holds.

Finally, for any initial value $x_{0}, y_{0} \in\left[\theta, v_{0}\right], x_{0} \leq y_{0}$, by constructing the iterative sequences

$\left\{\begin{array}{l}x_{n}(t)=f_{1}\left(t, x_{n-1}(t), y_{n-1}(t)\right)+\int_{0}^{t} g_{1}\left(s, x_{n-1}(s), y_{n-1}(s)\right) \mathrm{d} s, \\ y_{n}(t)=f_{2}\left(t, y_{n-1}(u), x_{n-1}(t)\right)+\int_{0}^{t} g_{2}\left(s, y_{n-1}(s), x_{n-1}(s)\right) \mathrm{d} s,\end{array}\right.$

one has $\left\|x_{n}-u^{*}\right\| \longrightarrow 0,\left\|y_{n}-u^{*}\right\| \longrightarrow 0(n \longrightarrow \infty)$, and for any $\alpha \in(0,1)$, there exists a natural number $n_{0}$ which satisfies as $n \geq n_{0}$, the following is obtained:

$$
\left\{\begin{array}{l}
\left\|x_{n}-u^{*}\right\| \leq 2 N_{p} \alpha^{n}\left\|v_{0}-u_{0}\right\|, \\
\left\|y_{n}-u^{*}\right\| \leq 2 N_{p} \alpha^{n}\left\|v_{0}-u_{0}\right\| .
\end{array}\right.
$$

This completes the proof of Theorem 2 .

\section{The Application of Nonlinear Differential Equations}

In this section, the following nonlinear initial value problems of the differential equation are considered:

$$
\begin{cases}u^{\prime}(t)=f_{1}(t, u, v)+\int_{0}^{T} g_{1}(s, u, v) \mathrm{d} s, & u(0)=u_{0}, \\ v^{\prime}(t)=f_{2}(t, u, v)+\int_{0}^{T} g_{2}(s, u, v) \mathrm{d} s, & v(0)=v_{0},\end{cases}
$$

where $f_{i}, g_{i} \in C\left[I \times \mathbf{R}_{+} \times \mathbf{R}_{+}, \mathbf{R}_{+}\right], i=1,2, I=[0, T]$, and $E$ is a real Banach space with norm $\|\cdot\|$.

For convenience, the following assumptions are made:

$\left(C_{1}\right)$ There exists the nonnegative bounded integrable functions $a(t), b(t), c(t), d(t)$ which satisfy

$$
\begin{aligned}
& f_{2}(t, u, \theta) \leq a(t) u+b(t), \\
& g_{2}(t, u, \theta) \leq c(t) u+d(t) .
\end{aligned}
$$

$\left(C_{2}\right)$ There exists constant $M>0$, for any $u, v \in E, u \leq v$, which satisfies

$$
\begin{aligned}
& f_{i}(t, v, u)-f_{i}(t, u, v) \geq-M(v-u), \\
& g_{i}(t, v, u)-g_{i}(t, u, v) \geq 0, \quad(i=1,2) .
\end{aligned}
$$

$\left(C_{3}\right)$ For any $u, v \in E, u \leq v$, the following is satisfied:

$$
\begin{aligned}
-M(v-u) & \leq f_{2}(t, v, u)-f_{1}(t, u, v) \leq c(t)(v-u), \\
0 & \leq g_{2}(t, v, u)-g_{1}(t, u, v) \leq a(t)(v-u) .
\end{aligned}
$$

$$
\left(C_{4}\right) \int_{0}^{T} a(t)(v-u) \mathrm{d} r \int_{0}^{t} K(r, s) \mathrm{d} s<e^{M t}, \forall t \in I .
$$

Then, the following theorem is obtained.

Theorem 3. Let $P$ be a normal cone in E. Suppose that conditions $\left(C_{1}\right)-\left(C_{4}\right)$ hold. Then, there exists a unique solution of equation (48) $\left(u^{*}, u^{*}\right)$, and there are iterative sequences converging to the unique solution, and corresponding error estimates are given.

Proof. Firstly, differential equation (48) is turned into integral equations. For any fixed $\eta \in C^{1}[J, E]$, the following one-order linear ordinary differential initial value problems in Banach spaces are investigated:

$$
\begin{cases}u^{\prime}=f_{1}(t, \eta, \eta)-M(u-\eta)+\int_{0}^{T} K(t, s) g_{1}(s, \eta, \eta) \mathrm{d} s, & u(0)=u_{0} \\ u^{\prime}=f_{2}(t, \eta, \eta)-M(u-\eta)+\int_{0}^{T} K(t, s) g_{2}(s, \eta, \eta) \mathrm{d} s, & u(0)=u_{0} .\end{cases}
$$


It is easy to know that $(u, u) \in C^{1}[I, E] \times C^{1}[I, E]$ is a solution of (52) if and only if $(u, u)$ is a solution of the following integral equations:

$$
\begin{aligned}
u(t)= & e^{-M t}\left[u_{0}+\int_{0}^{T} g_{1}(r, \eta(r), \eta(r)) \mathrm{d} r \int_{0}^{t} K(s, r) \mathrm{d} s\right] \\
& +e^{-M t} \int_{0}^{t} e^{M s}\left[f_{1}(s, \eta(s), \eta(s))+M \eta(s)\right] \mathrm{d} s, \\
u(t)= & e^{-M t}\left[u_{0}+\int_{0}^{T} g_{2}(r, \eta(r), \eta(r)) \mathrm{d} r \int_{0}^{t} K(s, r) \mathrm{d} s\right] \\
& +e^{-M t} \int_{0}^{t} e^{M s}\left[f_{2}(s, \eta(s), \eta(s))+M \eta(s)\right] \mathrm{d} s .
\end{aligned}
$$

Next, the operator $A, B: C^{1}[I, E] \times C^{1}[I, E] \longrightarrow$ $C^{1}[I, E]$ is defined as the following:

$$
\begin{aligned}
A(\eta, \eta)= & e^{-M t}\left[u_{0}+\int_{0}^{T} g_{1}(r, \eta(r), \eta(r)) \mathrm{d} r \int_{0}^{t} K(s, r) \mathrm{d} s\right] \\
& +e^{-M t} \int_{0}^{t} e^{M s}\left[f_{1}(s, \eta(s), \eta(s))+M \eta(s)\right] \mathrm{d} s, \\
B(\eta, \eta)= & e^{-M t}\left[u_{0}+\int_{0}^{T} g_{2}(r, \eta(r), \eta(r)) \mathrm{d} r \int_{0}^{t} K(s, r) \mathrm{d} s\right] \\
& +e^{-M t} \int_{0}^{t} e^{M s}\left[f_{2}(s, \eta(s), \eta(s))+M \eta_{(s)}\right] \mathrm{d} s .
\end{aligned}
$$

Obviously, $(\eta, \eta)$ is a solution of (48) if and only if

$$
\left\{\begin{array}{l}
\eta=A(\eta, \eta), \\
\eta=B(\eta, \eta) .
\end{array}\right.
$$

Next, similar to the proof of Theorem 2, it is tested whether the operators $A$ and $B$ satisfy the whole condition of Theorem 1 from conditions $\left(C_{1}\right)-\left(C_{4}\right)$. Therefore, the result of Theorem 3 is obtained from Theorem 1 .

\section{Data Availability}

The data used to support the findings of this study are available from the corresponding author upon request.

\section{Conflicts of Interest}

The author declares that there are no conflicts of interest regarding the publication of this paper.

\section{Authors' Contributions}

The author read and approved the final manuscript.

\section{Acknowledgments}

The author was supported by the Project of National Social Science Fund of China (NSSF) (18BTY015) and the Shandong Province Higher Educational Science and Technology Program (J16LI01).

\section{References}

[1] D. Guo and V. Lakshmikantham, "Coupled fixed points of nonlinear operators with applications," Nonlinear Analysis: Theory, Methods \& Applications, vol. 11, no. 5, pp. 623-632, 1987.

[2] D. J. Guo, "Extremal solutions of nonlinear Fredholm integral equations in ordered banach spaces," Northeastern Mathematical Journal, vol. 7, no. 4, pp. 416-423, 1991.

[3] J. X. Sun and L. S. Liu, "Iterative method for coupled quasisolution of mixed monotone operator equations," Applied Mathematics and Computation, vol. 52, pp. 301-308, 1992.

[4] L. S. Liu, "Iterative method for solutions and coupled quasisolution of nonlinear fredholm integral equations in ordered banach spaces," Indian Journal of Pure and Applied Mathematics, vol. 27, no. 10, pp. 959-972, 1996.

[5] Z. T. Zhang, "The fixed point theorem of mixed monotone operator and applications," Acta Mathematica Sinica, vol. 41, no. 6, pp. 1121-1126, 1998, in Chinese.

[6] H. Su, "The solutions of mixed monotone fredholm-type integral equations in Banach spaces," Discrete Dynamics in Nature and Society, vol. 2013, Article ID 604105, 7 pages, 2013.

[7] D. J. Guo, Nonlinear Functional Analysis, Jinan, Shandong Science and Technology Press, Jinan, China, Second edition, 1985.

[8] D. Guo, "Initial value problems for second-order integrodifferential equations in Banach spaces," Nonlinear Analysis: Theory, Methods \& Applications, vol. 37, no. 3, pp. 289-300, 1999.

[9] H. Zhang, Y. Li, and J. Xu, "Positive solutions for a system of fractional integral boundary value problems involving hadamard-type fractional derivatives," Complexity, vol. 2019, Article ID 2671539, 11 pages, 2019.

[10] H. R. Marasi, H. Afshari, and M. Daneshbastam, "Fixed points of mixed monotone operators for existence and uniqueness of nonlinear fractional differential equations," Journal of Contemporary Mathematical Analysis, vol. 52, p. 8C13, 2017.

[11] X. Zhang, Y. Wu, and L. Caccetta, "Nonlocal fractional order differential equations with changing-sign singular perturbation," Applied Mathematical Modelling, vol. 39, no. 21, pp. 6543-6552, 2015.

[12] W. Cheng, J. Xu, and Y. Cui, "Positive solutions for a class of fractional difference systems with coupled boundary conditions," Advances in Difference Equations, vol. 2019, no. 1, 2019.

[13] X. Zhang, L. Liu, and Y. Wu, "Multiple positive solutions of a singular fractional differential equation with negatively perturbed term," Mathematical and Computer Modelling, vol. 55, no. 3-4, pp. 1263-1274, 2012.

[14] K. Wu, Q. Wang, and K. Guan, "Iterative method and convergence analysis for a kind of mixed nonlinear VolterraFredholm integral equation," Applied Mathematics and Computation, vol. 225, pp. 631-637, 2013.

[15] T. Qi, Y. Liu, and Y. Cui, "Existence of solutions for a class of coupled fractional differential systems with nonlocal boundary conditions," Journal of Function Spaces, vol. 2017, Article ID 6703860, 9 pages, 2017.

[16] D. Wardowski, "Mixed monotone operators and their application to integral equations," Journal of Fixed Point Theory and Applications, vol. 19, no. 2, pp. 1103-1117, 2017.

[17] T. Qi, Y. Liu, and Y. Zou, "Existence result for a class of coupled fractional differential systems with integral boundary value conditions," The Journal of Nonlinear Sciences and Applications, vol. 10, no. 7, pp. 4034-4045, 2017. 
[18] M. H. Daliri and J. Saberi-Nadjafi, "Improved variational iteration method for solving a class of nonlinear Fredholm integral equations," SeMA Journal, vol. 76, no. 1, pp. 65-77, 2019.

[19] Z. Zhao, "Existence and uniqueness of fixed points for some mixed monotone operators," Nonlinear Analysis: Theory, Methods \& Applications, vol. 73, no. 6, pp. 1481-1490, 2010.

[20] X. Zhang, L. Liu, and Y. Wu, "Global solutions of nonlinear second-order impulsive integro-differential equations of mixed type in Banach spaces," Nonlinear Analysis: Theory, Methods \& Applications, vol. 67, no. 8, pp. 2335-2349, 2007.

[21] A. E. Taylar and D. C. Lay, Introduction to Function Analysis, Elsevier, New York, NY, USA, Second edition, 1980.

[22] X. Zhang, L. Liu, Y. Wu, and Y. Cui, "New result on the critical exponent for solution of an ordinary fractional differential problem," Journal of Function Space, vol. 2017, Article ID 3976469, 4 pages, 2017.

[23] C. Chen, X. Zhang, G. Zhang, and Y. Zhang, "A two-grid finite element method for nonlinear parabolic integro-differential equations," International Journal of Computer Mathematics, vol. 96, no. 10, pp. 2010-2023, 2019. 